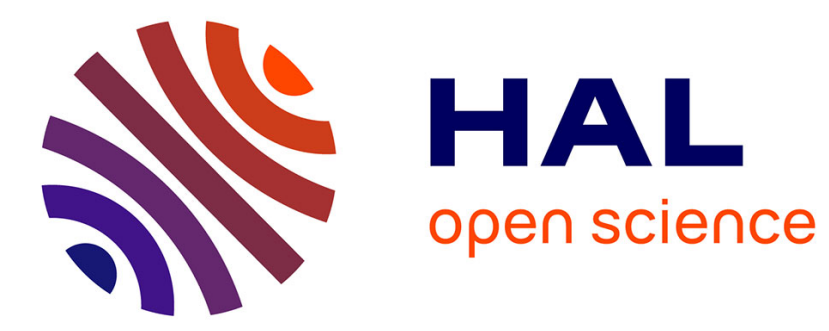

\title{
Real-Time Estimation in a Turbulent Jet Using Multiple-Input-Multiple-Output Transfer Functions
}

Igor Maia, Peter Jordan, Eduardo Martini, André Cavalieri, Aaron Towne, Lutz Lesshafft, Oliver Schmidt

\section{- To cite this version:}

Igor Maia, Peter Jordan, Eduardo Martini, André Cavalieri, Aaron Towne, et al.. Real-Time Estimation in a Turbulent Jet Using Multiple-Input-Multiple-Output Transfer Functions. 25th AIAA/CEAS Aeroacoustics Conference, May 2019, Delft, Netherlands. 10.2514/6.2019-2535 . hal-02378532

\section{HAL Id: hal-02378532 \\ https://hal.science/hal-02378532}

Submitted on 28 Nov 2019

HAL is a multi-disciplinary open access archive for the deposit and dissemination of scientific research documents, whether they are published or not. The documents may come from teaching and research institutions in France or abroad, or from public or private research centers.
L'archive ouverte pluridisciplinaire HAL, est destinée au dépôt et à la diffusion de documents scientifiques de niveau recherche, publiés ou non, émanant des établissements d'enseignement et de recherche français ou étrangers, des laboratoires publics ou privés. 


\title{
Real-Time Estimation in a Turbulent Jet Using Multiple-Input-Multiple-Output Transfer Functions
}

\author{
Igor A. Maia*, Peter Jordan ${ }^{\dagger}$, Eduardo Martini ${ }^{\ddagger}$, André V. G. Cavalieri ${ }^{\S}$, Aaron Towne ${ }^{\Uparrow}$ Lutz \\ Lesshafft $\|$,Oliver Schmidt**
}

In this work we investigate the use of multiple-input, multiple-output (MIMO) transfer functions obtained empirically from a large-eddy simulation of a turbulent jet. We compare the MIMO performance with single-input-single-output (SISO) transfer functions used in previous studies. The choice of sensor placement has been made based on results of linear stability analysis from the literature. The results show that MIMO transfer functions improve on SISO results where both single- and two-point statistics are concerned. It is also found that the number of sensors necessary to converge the estimates depends strongly on Strouhal number.

\section{Nomenclature}

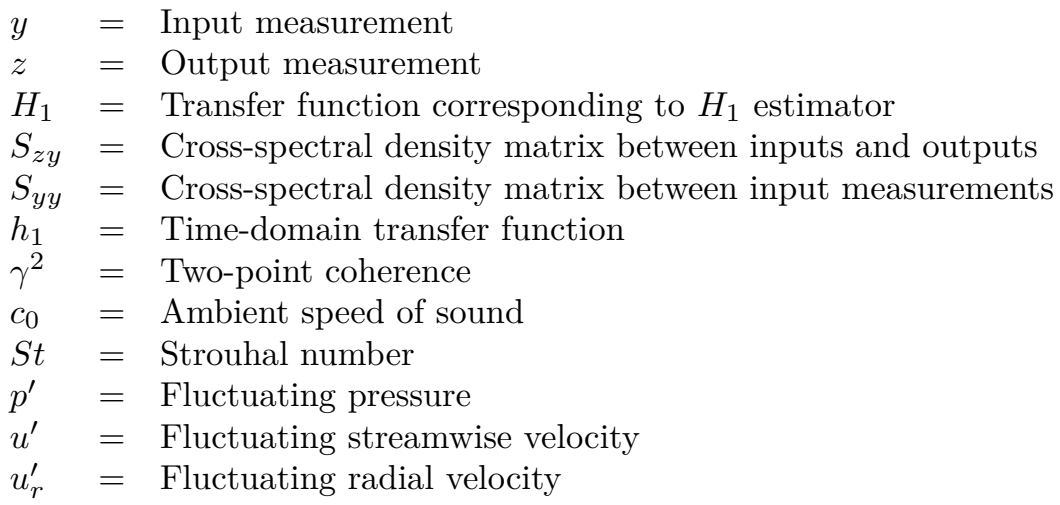

\section{Introduction}

Input-output analysis is increasingly used in the control of fluid systems, because it provides a framework in which to associate control design and reduced-order models, the latter being frequently based on hydrodynamic stability theory[1]. In this framework the frequency response of the system to given excitations is described by means of transfer functions, and this information is used to feed actuators via a control law so as to achieve a certain objective.

For a convectively unstable flow, the crucial step in this approach is the correct estimation of the evolution of flow disturbances as they are convected downstream. Therefore, a model that provides an accurate description of the convective flow dynamics is required. The use of reduced-order models based on linear stability theory for the control of open flows has been extensively revised by [2. Galerkin-based, reduced-order models have also been used in control theory. They rely on the projection of the linearised Navier-Stokes

\footnotetext{
*PhD student, PPrime Institute - Université de Poitiers-ENSMA. Poitiers, 86036, France.

${ }^{\dagger}$ Research Scientist, PPrime Institute - Université de Poitiers-ENSMA. Poitiers, 86036, France.

‡PhD Student, Instituto Tecnológico de Aeronáutica, São José dos Campos, Brazil.

$\S$ Assistant Professor, Instituto Tecnológico de Aeronáutica, São José dos Campos, Brazil.

ฯ Assistant Professor, Department of Mechanical Engineering, University of Michigan, Ann Arbor, MI,USA.

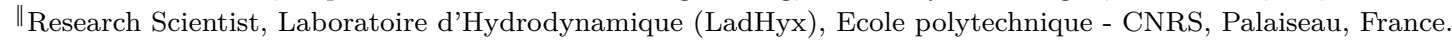

**Assistant Professor, Department of Mechanical and Aerospace Engineering, University of California San Diego, CA, USA.
} 
equation onto a basis, which results in a state-space equation. Proper Orthogonal Decomposition (POD) modes [3, 4, balanced modes [5, 6] and global eigenmodes [7, 8, have been used as such bases.

However, as pointed out by [9], Galerkin-based methods for control possess a number of limitations. For instance, the performance of such models depends on the observability of the associated modes from measurements, which may not be the case for POD modes. Moreover, the projections usually rely on a priori assumptions about the sources of ambient noise on the state variables, which should be taken into account by the model; if the true noise sources are different from the modelled ones, the controller may present stability issues.

An alternative to model-based control is system identification [9 11, which is a data-based technique that establishes a link between input and output through direct observations. System identification is particularly interesting for experimental applications, because the effect of external noise is implicitly embedded in the determination of the transfer function. In [9], an auto-regressive, moving-average, exogenous (ARMAX) model was used to perform estimation and control in a simulation of a flow over a backward-facing step, with significant reductions in turbulent kinetic energy.

For a turbulent jet, it has been shown in [12] that the space-time evolution of disturbances can be successfully predicted by two methods of transfer function identification: the parabolised stability equations (PSE) and a direct identification based on the cross-spectral density of input and output measurements. The former is a model approach based on first principles, and depends only on the mean flow; the latter is empirical and depends on measurements of fluctuating flow quantities. The authors used a single-input configuration and the results indicated that both methods perform equally well and provide good estimates when input and output are separated by a few jet diameters. Such single-input-single-output transfer functions based on PSE have later been used to perform closed-loop control in an incompressible shear-layer [13, 14]. However, the agreement between estimates and measurements deteriorates if input and outputs are moved further apart. Moreover, single-input transfer functions have the disadvantage of not being able to predict higher-order dynamics, such as jitter [15], which is manifest in the two-point coherence decay and is an important trait for sound radiation [16].

A method for estimating unknown two-point space-time statistics from a set of measurements has been developed in [17. The method is based on the resolvent framework [18, in which non-linear effects are interpreted as input forcing that drives linear dynamics via the resolvent operator of the linearised NavierStokes operator. In a model problem, based on the Ginzburg-Landau equation, it is shown that it is possible to improve estimates of power spectral density and cross-spectral density with the addition of input probes, which amounts to using multiple resolvent modes in the estimation of the forcing term. This method has been applied in a turbulent channel flow in [19], with good estimates of velocity energy spectra and autocorrelations near the wall.

The objective of this work is to explore how SISO estimation can be iproved by the introduction of multiple sensors. We are interested in the evolution of both disturbance amplitude and higher-order activity, manifest in the two-point coherence of the disturbance field. We use a large-eddy simulation (LES) database for a Mach $M a=0.4$, Reynolds number $R e=4.5 \times 10^{5}$, jet, performed using the compressible flow solver "Charles" 20]. The empirical approach is conceptually similar to the resolvent-based one used in [19]. However, the resolvent-based approach involves one intermediate step of inferring the forcing term from the set of available measurements; in the empirical framework, this step is implicit in the determination of transfer functions from the measurements.

An important question then arises: where to place the sensors in order to improve the predictions? Linear stability analysis can be used as a physics-based framework in which to guide sensor placement prior to the application of optimization algorithms. It has been previously shown that homogeneous linear stability models (such as PSE) can describe the evolution of flow disturbances up to the point where the Kelvin-Helmholtz $(\mathrm{KH})$ mode becomes neutrally stable [21-23]. Further downstream the agreement between homogeneous linear models and experiments breaks down; the results of [12] also show a deterioration of the estimates using transfer functions based on PSE in the region where the KH mode is unstable.

It has been shown in [24], by means of a locally parallel, spatial transient growth analysis, that a way to correct the discrepancies of the linear models is to account for the contribution of the stable modes, that are important due to the non-normality of the linearised Navier-Stokes operator. The study revealed that, concerning both the growth of disturbances and the two-point coherence, the spatial evolution of disturbances involves linear combination of the stable modes, in a manner that may be mimicked by a MIMO model. 
These stable modes have extended spatial support in the shear-layer, but have different radial organisation. Based on these observations, we radially distribute the sensors in the shear-layer of the jet to perform the MIMO analysis; the idea being to pick up information from different stable modes so as to improve the estimates of the SISO transfer functions which, by construction, can only carry information of a single-mode.

The remainder of the paper is organised as follows: in section \&III the methodology for computing the transfer functions from flow data is shown. In section $\$ I V$ we assess the performance of the two methods in predicting power spectral densities and two-point coherence of the disturbances. Finally, in section $\S \mathrm{V}$ we summarise the main conclusions of the study.

\section{Methodology}

A time-invariant dynamical system can be represented by [25, 26]

$$
z=H y+\epsilon,
$$

where $y, z$ are inputs and responses (outputs), $H$ is the transfer function and $\epsilon$ is the error. We have used the $H_{1}$ estimator as a transfer function, which assumes that the error, $\epsilon$, is uncorrelated with the input, $y$. For the case of a multiple-input-multiple-output system, the $H_{1}$ estimator is given, in the frequency domain, by

$H_{1}(\omega)=S_{z y}(\omega) S_{y y}^{-1}(\omega)=\left[\begin{array}{cccc}S_{z_{1} y_{1}}(\omega) & S_{z_{1} y_{2}}(\omega) & \cdots & \left.S_{z_{1} y_{m}} \omega\right) \\ S_{z_{2} y_{1}}(\omega) & S_{z_{2} y_{2}}(\omega) & \cdots & S_{z_{2} y_{m}}(\omega) \\ \vdots & \vdots & \ddots & \vdots \\ S_{z_{n} y_{1}}(\omega) & S_{z_{n} y_{2}}(\omega) & \cdots & S_{z_{n} y_{m}}(\omega)\end{array}\right]\left[\begin{array}{cccc}S_{y_{1} y_{1}}(\omega) & S_{y_{1} x_{2}}(\omega) & \cdots & S_{y_{1} y_{m}}(\omega) \\ S_{y_{2} y_{1}}(\omega) & S_{y_{2} y_{2}}(\omega) & \cdots & S_{y_{2} y_{m}}(\omega) \\ \vdots & \vdots & \ddots & \vdots \\ S_{y_{m} y_{1}}(\omega) & S_{y_{m} x_{2}}(\omega) & \cdots & S_{y_{m} y_{m}}(\omega)\end{array}\right]^{-1}$

where $S_{z_{i} y_{k}}$ is the cross spectral density of the $k^{t h}$ input and the $i^{t h}$ output, and $S_{y_{i} y_{k}}$ is the cross spectral density of the $k^{t h}$ and $i^{t h}$ inputs. In the case of a single-input-single-output system, the expression reduces to

$$
H_{1}=S_{z y} / S_{y y} .
$$

A time-domain transfer function, $h_{1}(t)$, can be obtained through the inverse Fourier transform of $H_{1}$,

$$
h_{1}(t)=\frac{1}{2 \pi} \int_{-\infty}^{\infty} H_{1}(\omega) e^{-i \omega t} \mathrm{~d} \omega .
$$

Once the time-domain transfer function is obtained, the output $z(t)$ can be obtained by convolution of some input measurements with the causal part of $h_{1}(t)$,

$$
z(t)=\int_{0}^{\infty} h_{1}(t-\tau) y(\tau) \mathrm{d} \tau
$$

Equation 5 is used to estimate downstream flow disturbances in the jet based on measurements made at a given upstream position. Three quantities have been used to perform the estimation: pressure, streamwise velocity and radial velocity. All the estimations concern the axisymmetric azimuthal mode of the jet only. Throughout the paper we show estimations of the power spectral density of outputs, $S_{z z}$, and two-point coherence, defined, for different outputs $z_{i}$ and $z_{j}$, as

$$
\gamma_{i j}^{2}(\omega)=\frac{\left\langle S_{z_{i} z_{j}}(\omega)\right\rangle^{2}}{\left\langle S_{z_{i} z_{i}}(\omega)\right\rangle^{2}\left\langle S_{z_{j} z_{j}}(\omega)\right\rangle^{2}},
$$

where the symbol $\langle\cdot\rangle$ denotes ensemble average.

We use an LES database of a $M a=0.4$ turbulent jet generated using the flow solver "Charles" [20. The power- and cross-spectral density matrices have been computed using Welch's method. The jet is isothermal and synthetic turbulence is introduced in the nozzle to obtain a fully turbulent boundary layer. The simulation was run for a total time of 2000 acoustic time units, where the acoustic time is defined as $t c_{0} / D$, with $c_{0}$ is the ambient speed of sound and $D$ the jet diameter. The unstructured grid was interpolated to a cylindrical 
grid spanning $0 \leqslant x / D \leqslant 30,0 \leqslant r / D \leqslant 6,0 \leqslant \phi \leqslant 2 \pi$. This database has also been used by [27] to perform spectral proper orthogonal decomposition (SPOD) and resolvent analysis with a view to study the low-rank behaviour of the jet.

A preliminary analysis of SISO transfer functions was carried out to determine the Strouhal-number range in which input and output have a linear phase relation. For the two streamwise sensor positions described in section\$[V] a linear phase relation was observed up to Strouhal number $S t=1$, a deterioration occurring at higher St. For this reason, the time signals have been filtered using a 30th order low pass FIR filter with a cutoff Strouhal number of $S t_{c u t}=1$.

\section{Results}

Throughout the paper, we systematically compare estimates made using SISO and MIMO transfer functions with data from the LES. We perform estimations of the evolution of flow disturbances in the shear-layer in two different zones of the jet. In the first, the input sensors are situated at $x / D=2$, where the $\mathrm{KH}$ mode is spatially unstable for a range of Strouhal numbers. In the second, the sensors are situated at $x / D=6$, where the $\mathrm{KH}$ mode is stable [24]. Estimations are performed with outputs distributed over an extended streamwise region and along a line of constant radial position.

Different numbers of sensors are tested for the MIMO estimations. In section \$IV.A we show results obtained with 50 sensors distributed in the shear-layer and we compare with the estimates obtained using a single sensor. In section \$IV.B we analyse the convergence of the MIMO estimates with increasing number of sensors.

\section{A. SISO vs. MIMO estimation}

We consider first the region of unstable KH mode. Figure 1 shows the distribution of input and output sensors. Input sensors are radially distributed at $x / D=2$. The output sensors are distributed between $x / D=3$ and $x / D=5$ at a fixed radial position, $r / D=0.4$. The shading area shows the mean flow. The distribution of sensors with respect to the radial rms profiles of pressure, streamwise velocity and radial velocity is also shown. The MIMO transfer-function, given in equation 2, was computed separately for each variable. The SISO estimations were performed with the input located at the same radial position as the outputs, $r / D=0.4$, and each input-output pair was computed separately using equation 3 .

Figure 2 shows estimates of the time signals of fluctuating pressure, streamwise velocity and radial velocity given by MIMO and SISO transfer functions compared with the time signals from the LES. The output sensor is located at $x / D=3$. Pressure fluctuations have been non-dimensionalised as $p^{\prime}=p / \rho_{0} c_{o}^{2}$, where $\rho_{0}$ is the ambient density and $c_{0}$ the ambient sound speed, and velocities have been non-dimensionalised by the jet exit velocity, $U j$. We note that the SISO predictions are reasonably good, both in terms of amplitude and phase. Only minor improvements are observed in the time signals with the addition of other sensors in the MIMO model. However, a more pronounced difference can be seen by looking at the power spectral density (PSD) estimates, shown in Figure 3 as a function of Strouhal number for the same output position. It can be seen that the MIMO approach improves estimates over a wide range of Strouhal numbers, for the three variables tested. It is also interesting to note that around $S t=0.6$, at the peak of the spectrum, both methods perform equally well, and the estimates are remarkably good. The interpretation for this trend will be discussed in more detail in section \$IV.B

Figure 4 shows the estimates of two-point coherence between output sensors placed at $x / D=3$ and $x / D=4$. Here a striking difference can be seen between SISO and MIMO: in the SISO model, coherence between input and output is, by construction, equal to one. Therefore coherence decay between two points separated in the flow cannot be described [24]. However, the addition of the other sensors improves coherence predictions significantly, and the agreement between MIMO and LES data is good. The difference between SISO and MIMO to predict coherence decay can be further appreciated in figure 5 which shows coherence maps as a function of Strouhal number based on pressure measurements, for outputs located between $x / D=3$ and $x / D=5$, respectively at the beginning and the end of the red zone shown in figure 1. The reference point is at $x / D=3$. It can be observed that the MIMO model reproduces well the coherence map of the LES, for a wide range of Strouhal numbers, in contrast to the SISO, which yields unit coherence.

Figures 6. 9 show the same set of results for inputs and outputs placed in the stable KH zone. Estimations were again made in the shear-layer, at $r / D=0.4$. The same trends observed in the unstable zone also hold in 


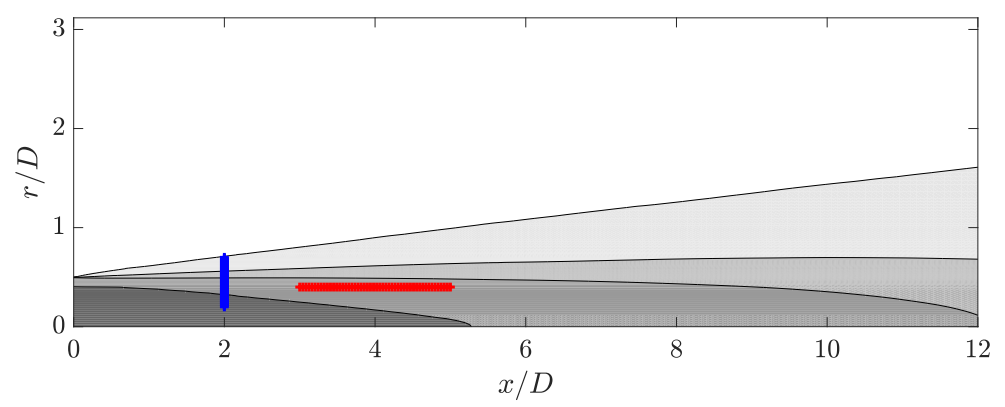

(a)

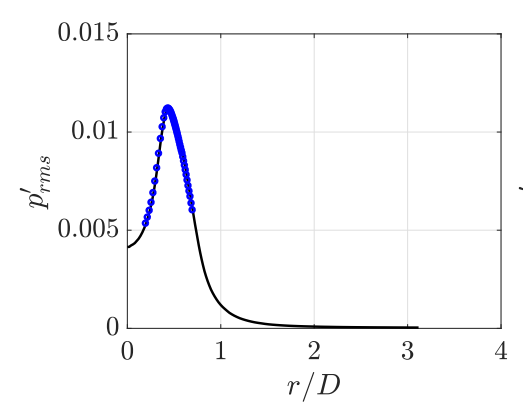

(b)

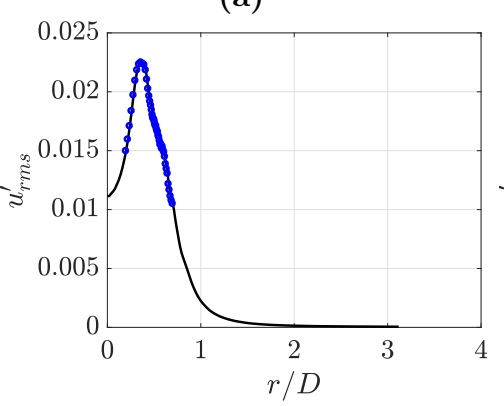

(c)

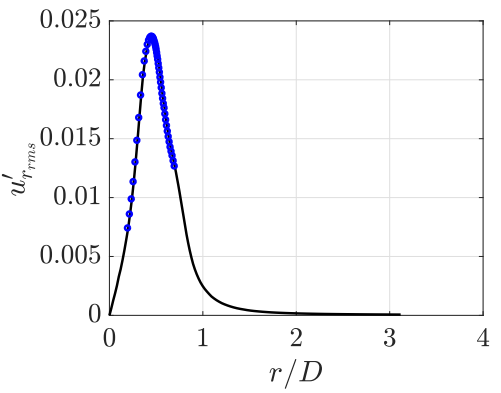

(d)

Fig. 1 Top: Distribution of input (blue) and output (red) points in the jet. Bottom: distribution of input sensors in the shear-layer: rms plots of pressure (left), streamwise velocity (middle) and radial velocity (right). Input sensors are at streamwise position $x / D=2$.

the stable zone: the MIMO transfer functions improve estimates of power spectral density over a broad range of the Strouhal number and reproduce the two-point coherence with globally reasonable accuracy. The dips in coherence seen at $S t>1$ for the SISO result in figures 3 and 8 are due to the fact that, at these Strouhal numbers, the phase of the transfer function deteriorates, as explained previously.

An important parameter in flow control is the distance between sensor and actuator. Estimation accuracy generally decreases with increasing separation distance between input and output [13, which imposes a limitation as to how far apart sensor and actuator can be set. It is thus interesting to assess the ability of SISO and MIMO transfer functions in the unstable and stable $\mathrm{KH}$ zones to predict the evolution of the disturbance amplitude with increasing separation distance between input and output.

Figure 10 shows predictions of power spectral density of pressure with increasing streamwise separation for two Strouhal numbers: $S t=0.6$, at the peak of the spectrum in the unstable KH zone, where the estimates are most accurate; and $S t=0.2$, at which the agreement between SISO and LES data is poorer at the unstable zone. Inputs are located at two different streamwise positions: $x / D=2$ (unstable KH zone) and $x / D=6$ (stable zone). Note that the MIMO model performs equally well in both zones and that estimates are quite good even for separation distances as high as 8 jet diameters. The predictions of the SISO model, on the other hand, have the tendency of gradually deteriorating when the output is moved further downstream. However, in the unstable zone at $S t=0.6$ (near the peak of the spectrum), SISO and MIMO provide estimates of similar accuracy for separations distances up to 3 jet diameters. This suggests that, for close separation distances, closed-loop control strategies based on single-input measurements, like the one applied in [13, may be successful if implemented in the unstable zone.

The results show that significant improvements in the estimation of flow disturbances in the jet can be achieved by MIMO transfer functions with sensors distributed in the shear-layer. We interpret these improvements as the effect of picking up information about different stable modes that have spatial support in the shear-layer; these modes have been shown, on one hand, to be important for the continued growth of disturbances in the zone where the $\mathrm{KH}$ mode becomes stable and, on the other, to provide a mechanism for coherence decay 24 . 


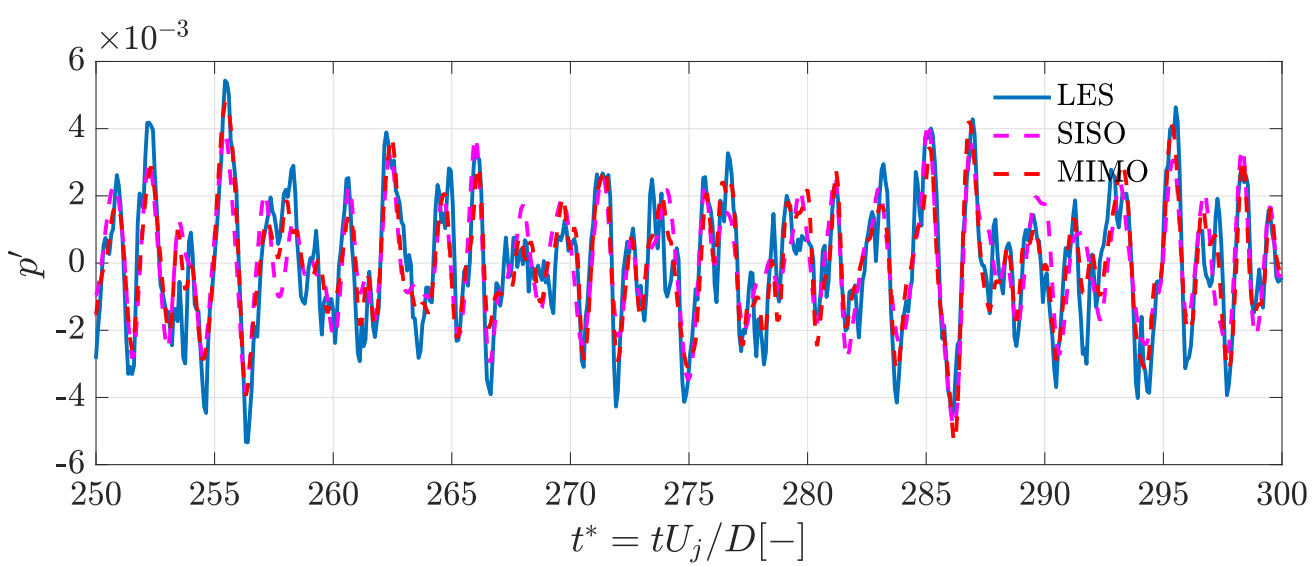

(a)

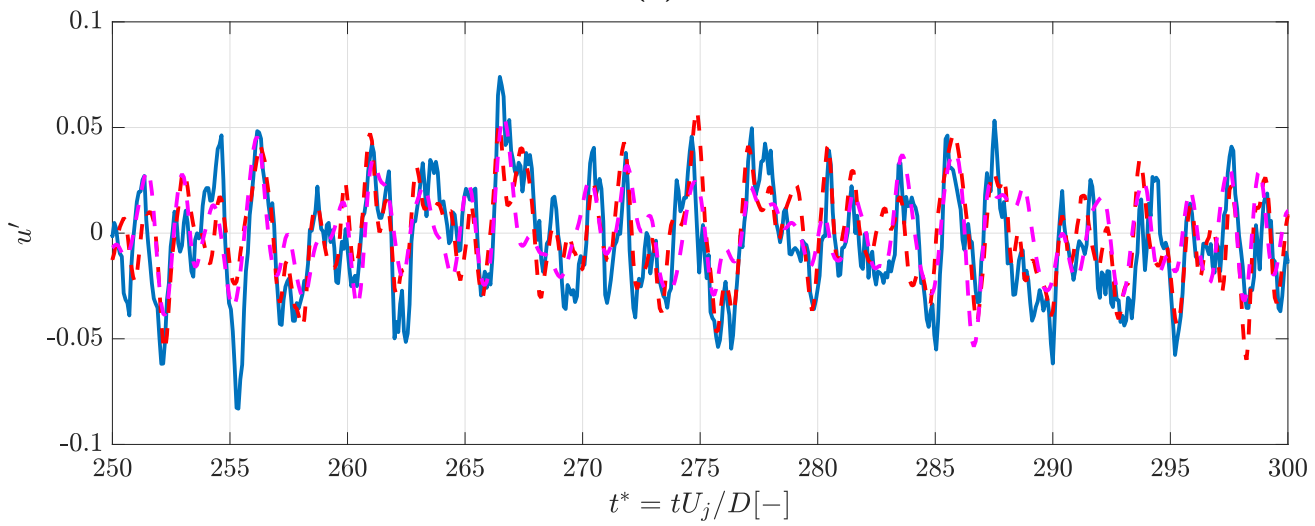

(b)

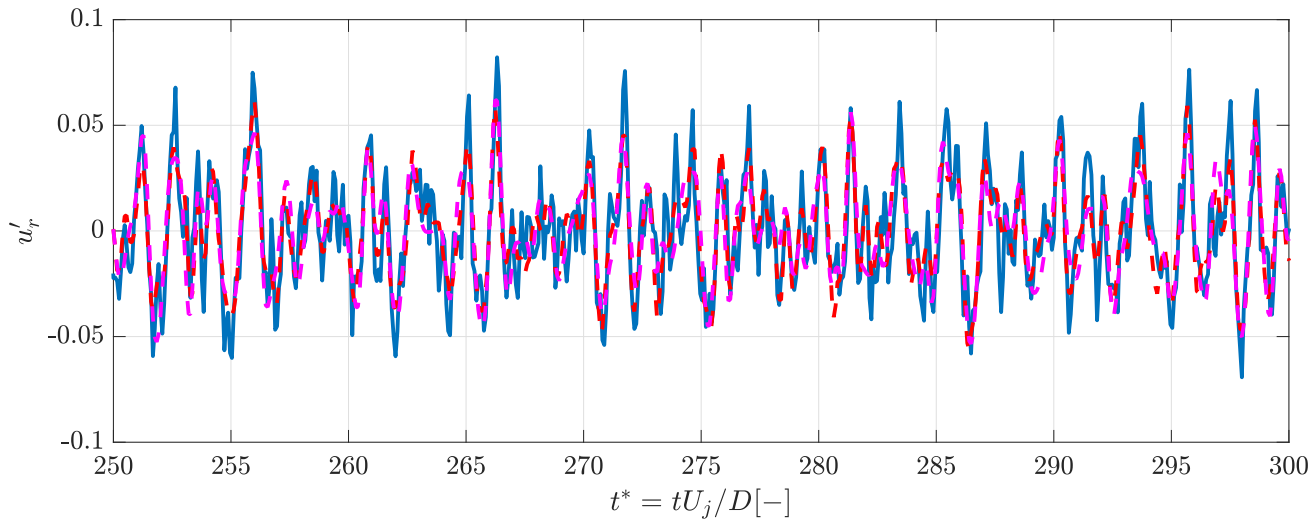

(c)

Fig. 2 Estimation of pressure (top), streamwise velocity (middle) and radial velocity (bottom) at $x / D=3$ by MIMO and SISO transfer functions based on input sensors placed at $x / D=2$. $t^{*}$ is a non-dimensional time.

\section{B. Convergence of MIMO estimates}

MIMO results presented above have been obtained with 50 sensors. We now assess the convergence of the MIMO estimates with increasing numbers of sensors distributed uniformly along the shear-layer, spanning a fixed radial width, e.g., $0.2 \leqslant r / D \leqslant 0.7$ for sensors placed at streamwise position $x / D=2$, and $0 \leqslant r / D \leqslant 0.8$ for sensors placed at $x / D=6$. For the PSD, the criterion to assess convergence is the squared error between LES data and estimate; for the two-point coherence, we simply use the difference 


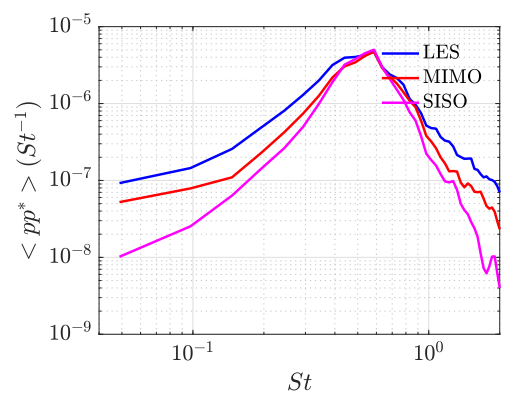

(a)

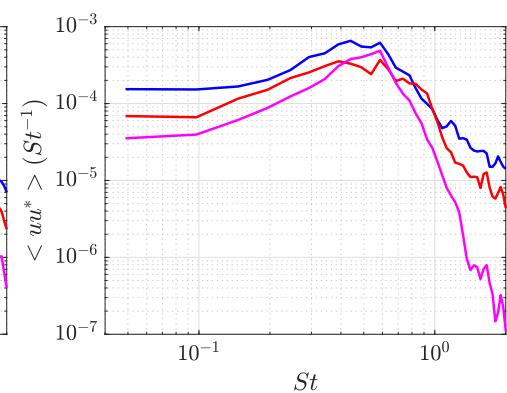

(b)

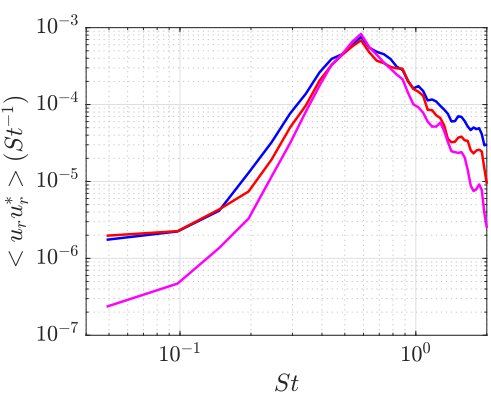

(c)

Fig. 3 Power spectrum density of pressure (left), streamwise velocity (middle) and radial velocity (right) estimated at $(x / D, r / D)=(3,0.4)$ from input measurements made at $x / D=2$.

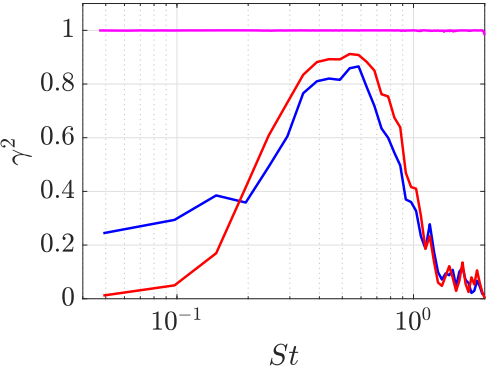

(a)

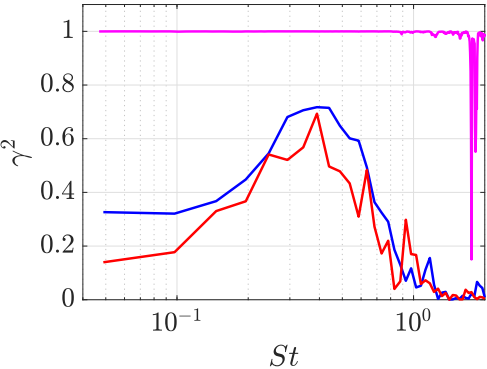

(b)

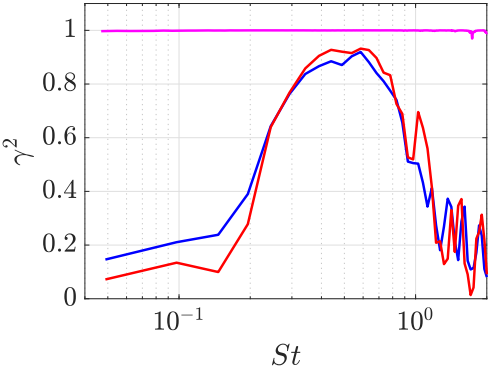

(c)

Fig. 4 Two-point coherence estimated between $(x / D, r / D)=(4,0.4)$ and $(x / D, r / D)=(3,0.4)$ based on pressure signal (left), streamwise velocity (middle) and radial velocity (right). Input sensor were placed at $x / D=2$. Color code is the same as in figure 3 .

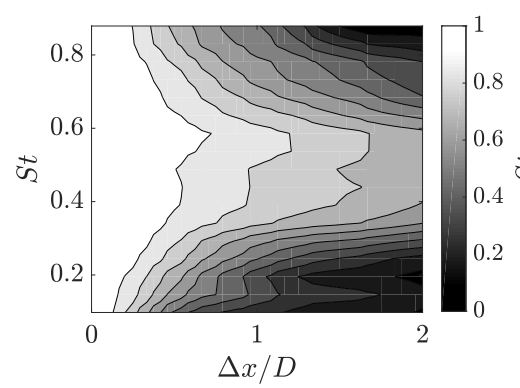

(a)

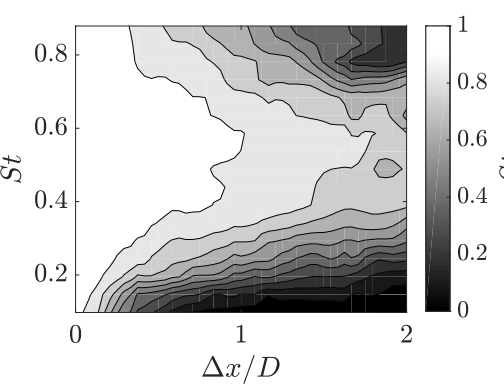

(b)

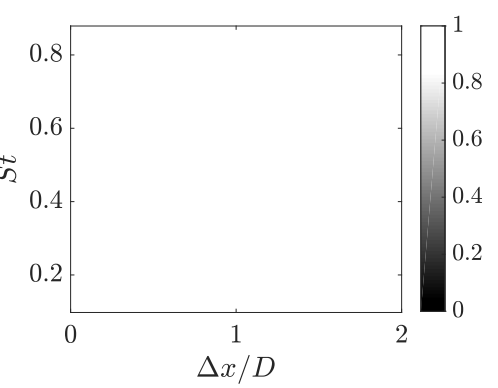

(c)

Fig. 5 Coherence maps based on pressure measurements between $x / D=3$ and $x / D=5$ for LES data (left), MIMO prediction (middle) and SISO prediction (right). Reference point is at $x / D=3$ and output sensors are located at radial position $r / D=0.4$.

$\Delta \gamma^{2}=\gamma_{L E S}^{2}-\gamma_{\text {est }}^{2}$ as a metric. The errors have been analysed in the frequency domain as a function of Strouhal number.

Figure 11 shows the convergence of the estimates of power spectral density and coherence in the unstable $\mathrm{KH}$ zone for different Strouhal numbers. In the convergence of power spectral density of pressure and radial velocity, two clear trends can be noticed: i) the smallest errors and faster convergence have been achieved at $S t=0.6$. At this Strouhal number, even a single sensor yields a very good estimate (less than $5 \%$ error.); ii) the greatest errors and slowest convergence have been observed at $S t=0.2$. These trends can be interpreted in light of recent results of resolvent analysis [27]29] for the same jet we consider. The gain spectrum of the resolvent operator performed by [27, 28] has revealed a large separation between the gains of the optimal 


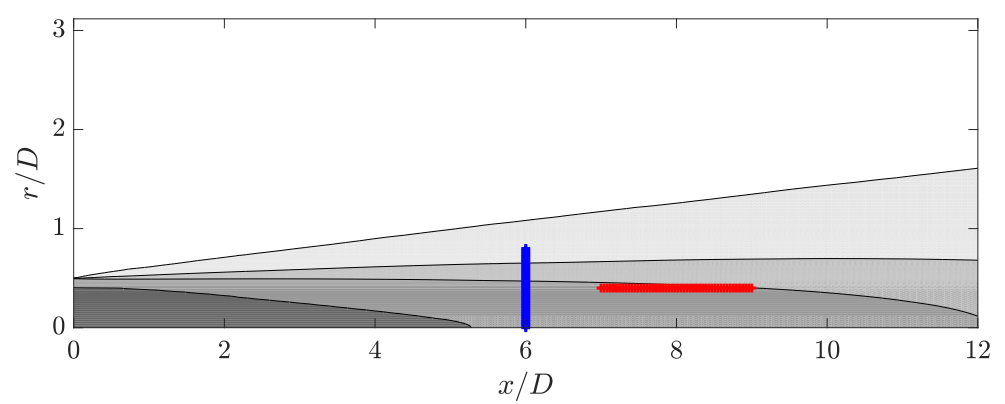

(a)

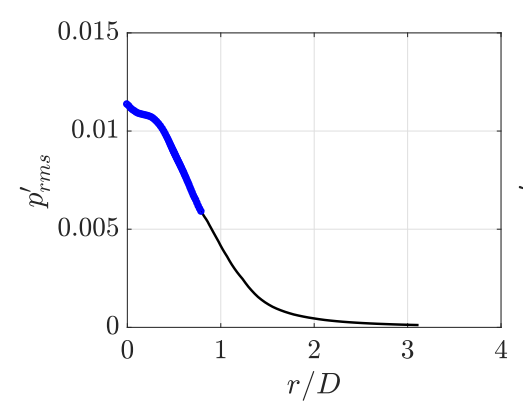

(b)

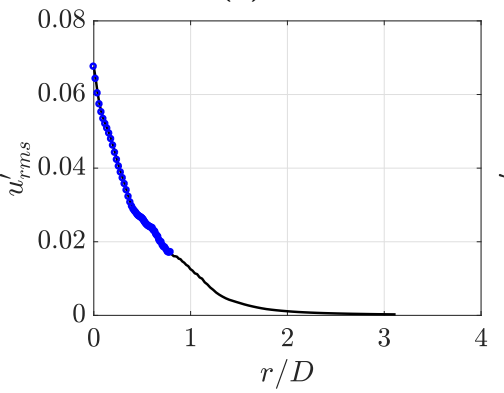

(c)

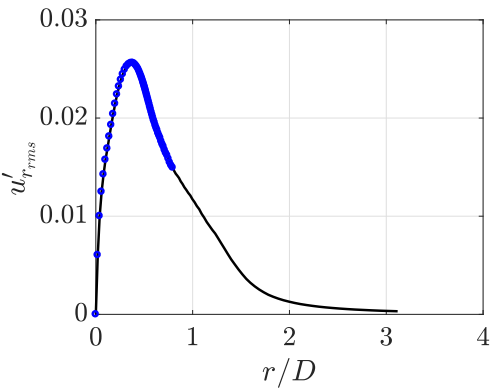

(d)

Fig. 6 Top: Distribution of input (blue) and output (red) points in the jet. Bottom: distribution of input sensors in the shear-layer: rms plots of pressure (left), streamwise velocity (middle) and radial velocity (right). Input sensors are at streamwise position $x / D=6$.

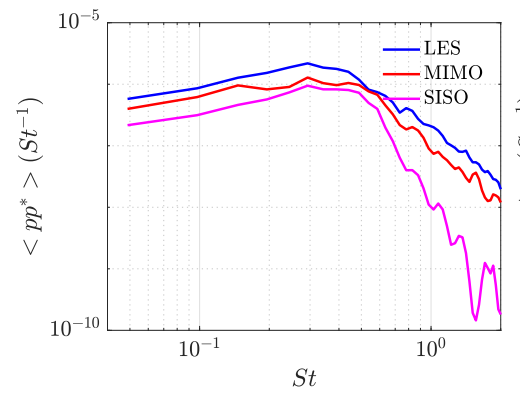

(a)

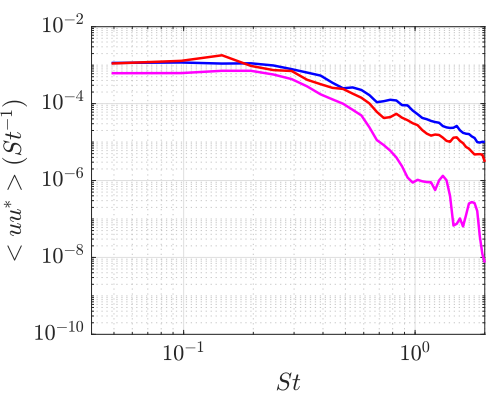

(b)

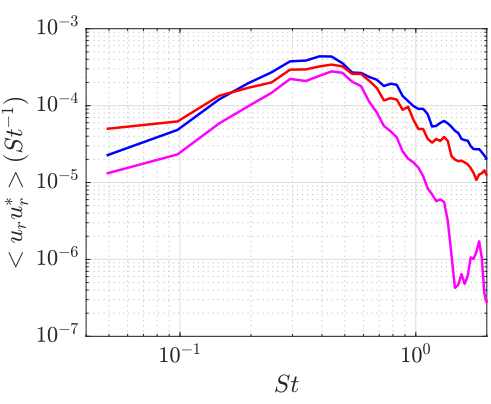

(c)

Fig. 7 Power spectrum density of pressure (left), streamwise velocity (middle) and radial velocity (right) estimated at $(x / D, r / D)=(7,0.4)$ from input measurements made at $x / D=6$.

and suboptimal modes in the range $0.3 \leqslant S t \leqslant 2$, with the largest gain separation being at $S t=0.6$ and the smallest one being at $S t=0.2$.

These large and low gain separations indicate, repectively, a low- and high-rank behaviours of the jet. This result has been confirmed by spectral orthogonal decomposition (SPOD [30]), whose gain also exhibit a large separation between optimal and suboptimals in the same region of the spectrum [27, 29] hence revealing a low-rank behaviour of the flow. This low-rank behaviour has been found to be more pronounced around $S t=0.6$; it is consistent with, and largely explains the results in figure 11. The high-rank behaviour at $S t=0.2$ is also consistent with the results of figure 11 for $S t=0.2$, which showed higher errors and slower convergence with increasing number of sensors.

These interpretations do not apply for streamwise velocity, which does not present a clear trend concerning the distribution of the error by Strouhal number. The oscillations seen in the convergence of the error in the PSD suggest a greater sensitivity of the estimates of streamwise velocity concerning sensor placement, which may be associated with the radial structure of the different stable modes. 


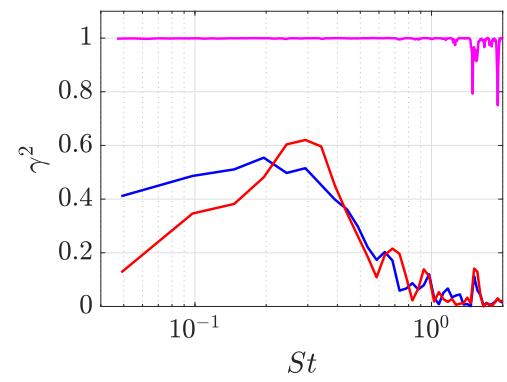

(a)

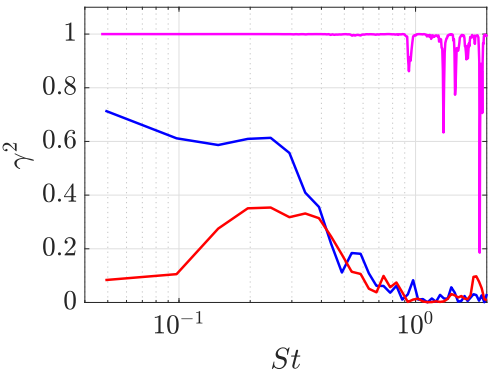

(b)

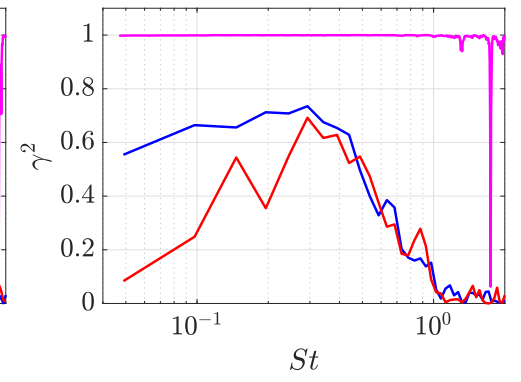

(c)

Fig. 8 Two-point coherence estimated between $(x / D, r / D)=(8,0.4)$ and $(x / D, r / D)=(7,0.4)$ based on pressure signal (left), streamwise velocity (middle) and radial velocity (right). Input sensor were placed at $x / D=6$. Color code is the same as in figure 3 .

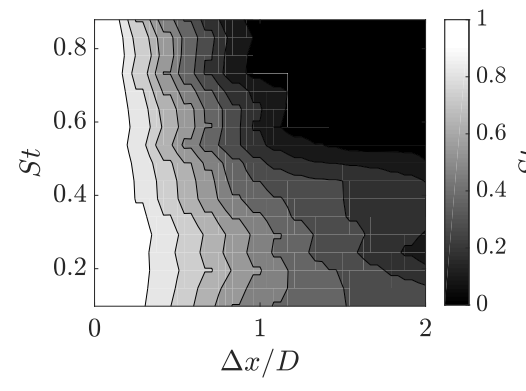

(a)

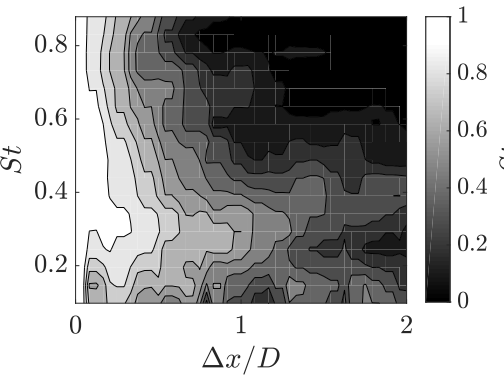

(b)

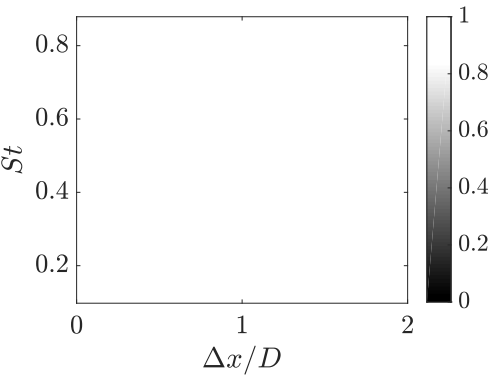

(c)

Fig. 9 Coherence maps based on pressure measurements between $x / D=7$ and $x / D=9$ for LES data (left), MIMO prediction (middle) and SISO prediction (right). Reference point is at $x / D=7$ and output sensors are located at radial position $r / D=0.4$.

Regarding two-point coherence, for pressure and radial velocity the smallest errors and fastest convergence were also observed at $S t=0.6$, as was the case for the PSD estimates. However, for streamwise velocity, analogously to what was observed in the convergence of the PSD, there is no clear trend with respect to Strouhal number.

Figure 12 shows the convergence of the estimates in the stable KH zone. Similar to what is observed in the unstable $\mathrm{KH}$ zone for pressure and radial velocity, the smallest errors and number of sensors in the estimate of PSD are obtained at $S t=0.6$, at the peak of the high gain separation. While this has been the case in the unstable $\mathrm{KH}$ zone, this is not the case within the stable zone. As a matter of fact, for streamwise velocity the smallest errors are found for $S t=0.2$, which is in the region of low rank separation. This disagreement reveals than a certain caution is necessary when using results of the global resolvent analysis to interpret local estimates. In this sense, a local analysis can also be instructive to shed some light on the discrepancies. Comparison of figures 11 and 12 reveals that in the stable KH zone both SISO and MIMO models perform better in the unstable KH zone and that the MIMO estimates concerning both PSD and coherence converge with less sensors.

\section{Conclusions}

We have used MIMO transfer functions identified empirically from an LES database with a view to improving estimates of SISO approches used in past studies [12, 13. The main conclusions can be summarised as follows:

- The estimation of single- and two-point statistics using empirical transfer functions can be improved with the addition of sensors radially distributed across the shear-layer of the jet, as information from multiple stable local modes may thus be incorporated in the estimation; 


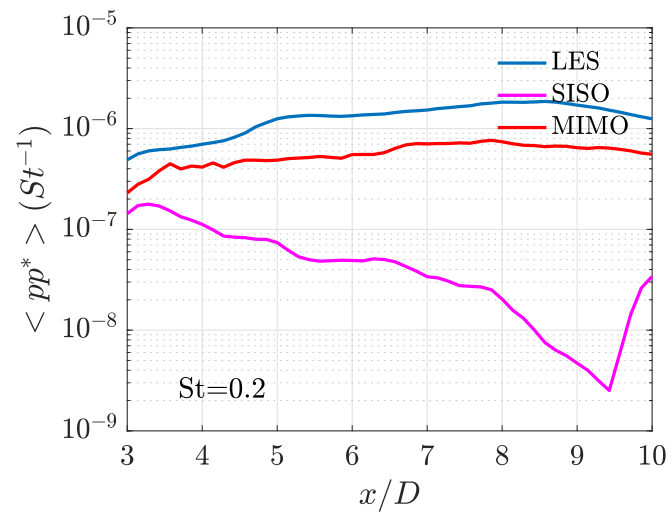

(a)

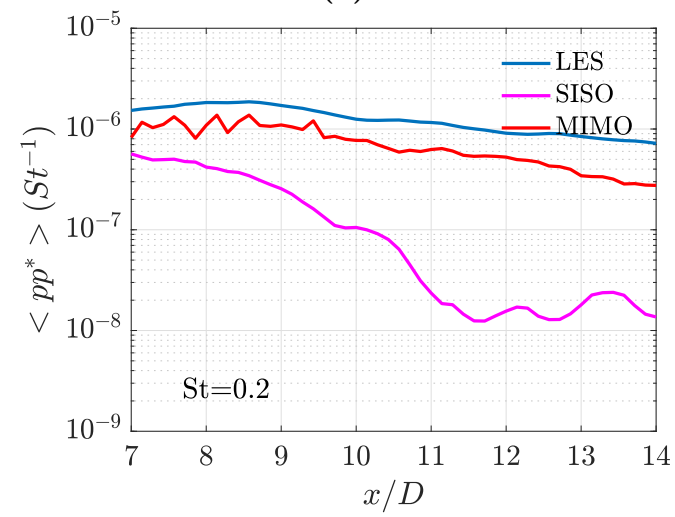

(c)

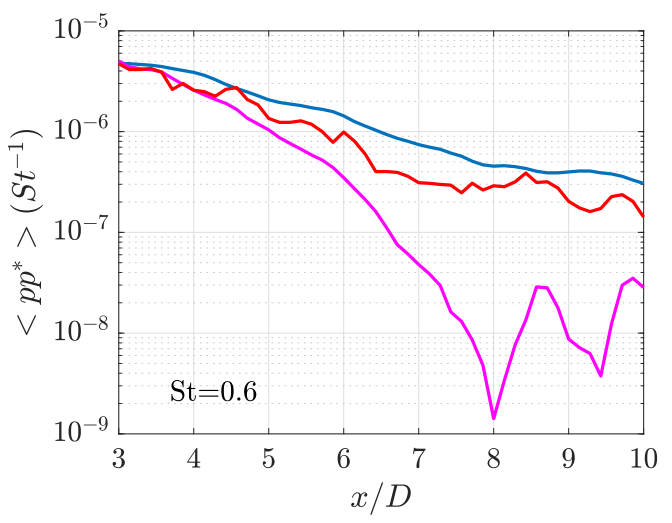

(b)

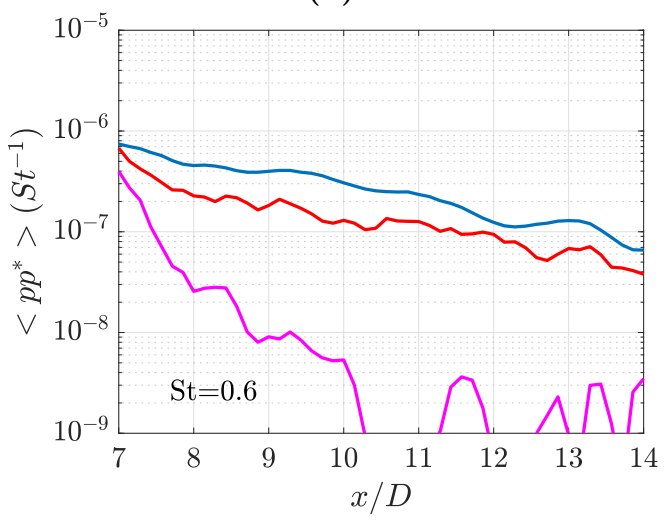

(d)

Fig. 10 Evolution of the power spectrum density of pressure with streamwise distance for two different Strouhal numbers. Top row: input sensors placed at $x / D=2$ (unstable KH zone) and outputs between $x / D=3$ and $x / D=10$. Bottom row: input sensors placed at $x / D=6$ (stable KH zone) and outputs between $x / D=7$ and $x / D=14$. Outputs are located at the line constant radial position, $r / D=0.4$.

- MIMO estimates of the streamwise evolution of disturbance amplitudes perform equally well in unstable and stable regions of the jet and provide good accuracy even for large separation distances between input and output. On the other hand, the SISO model is most accurate in the unstable zone where, for Strouhal numbers near the peak of the spectrum and small separation distances, its performance is similar to that of the MIMO model. This suggests that control strategies based on a single-input measurement may be appropriate in this zone;

- The convergence of the estimates with increasing number of sensors depends on Strouhal number and streamwise position. The trends can be understood, to a certain extent, in terms of the high- and low-rank wavepacket dynamics as predicted by global resolvent analyses. 


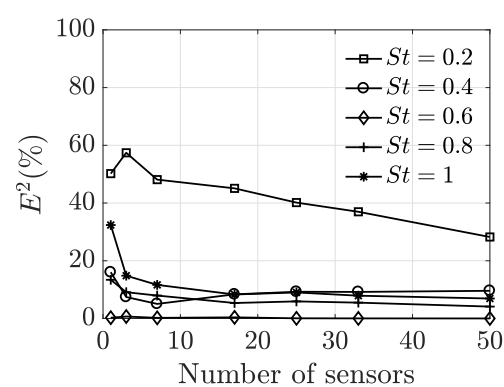

(a)

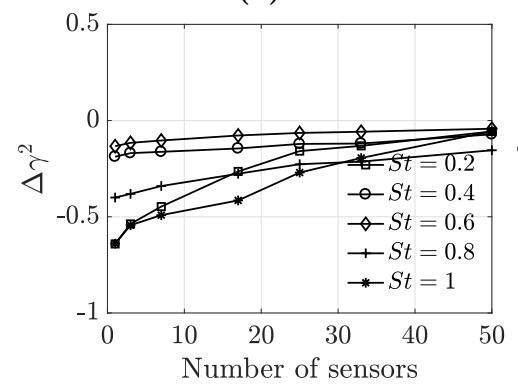

(d)

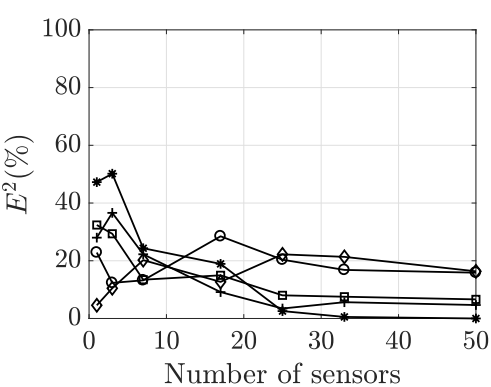

(b)

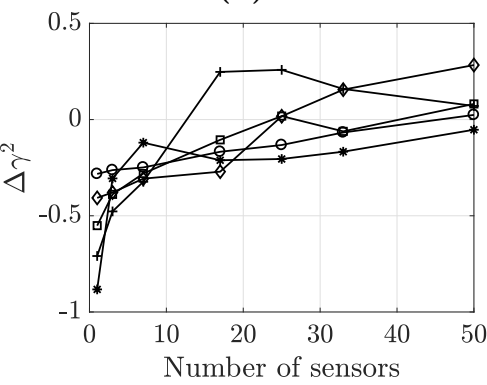

(e)

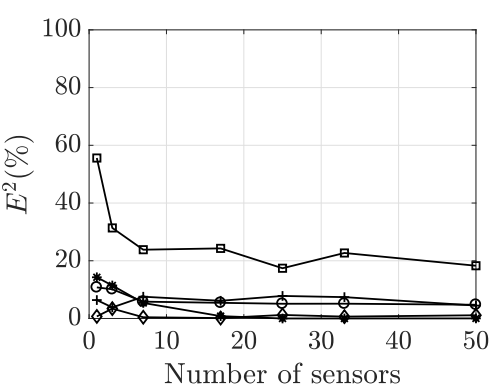

(c)

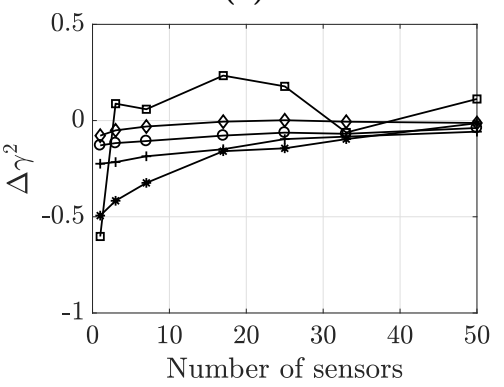

(f)

Fig. 11 Top row: convergence of the squared error of the power spectral density at $x / D=3$ with increasing number of points in MIMO estimate for different Strouhal numbers. Bottom row: convergence of two-point coherence estimate between $x / D=3$ and $x / D=5$. Left column: pressure; middle column: streamwise velocity; right column: radial velocity. Sensors have been placed at $x / D=2$.

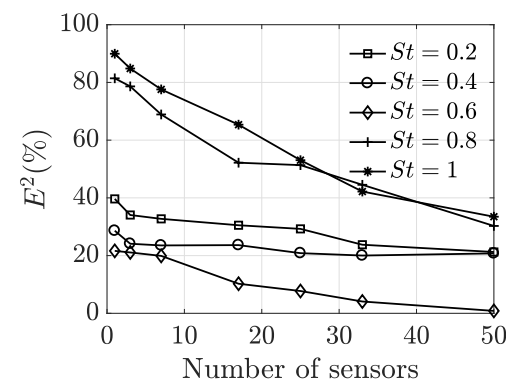

(a)

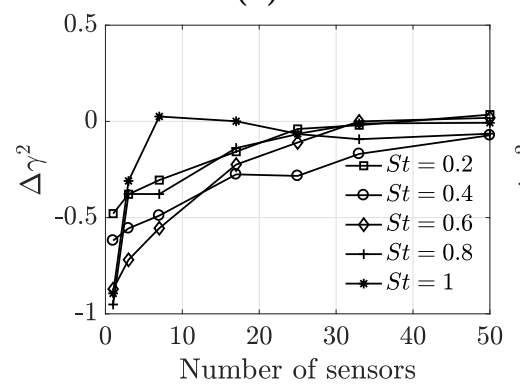

(d)

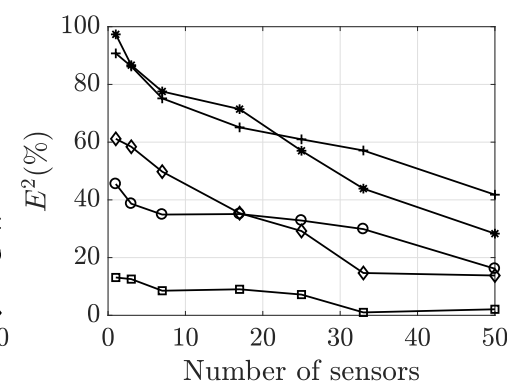

(b)

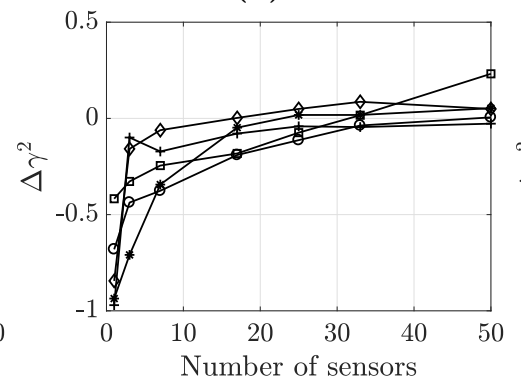

(e)

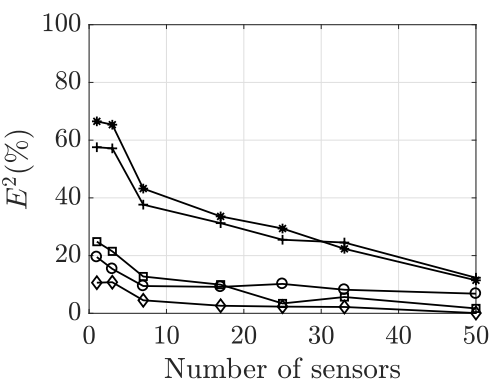

(c)

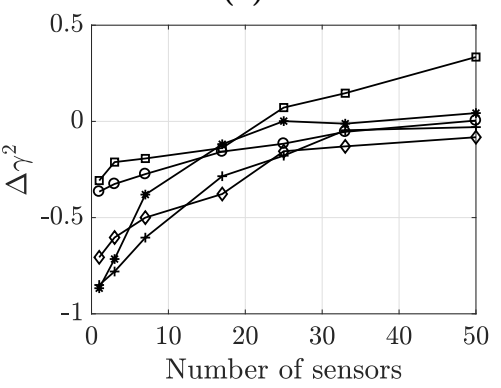

(f)

Fig. 12 Top row: convergence of the squared error of the power spectral density at $x / D=7$ with increasing number of points in MIMO estimate for different Strouhal numbers. Bottom row: convergence of two-point coherence estimate between $x / D=7$ and $x / D=9$. Left column: pressure; middle column: streamwise velocity; right column: radial velocity. Sensors have been placed at $x / D=6$. 


\section{Acknowledgments}

This work as supported by CNPq- National Council of Scientific and Technological Development - Brazil through the Science without Borders Program under grant number 200676/1025-6 and also through project A073/2013.

\section{References}

[1] Bagheri, S., Henningson, D. S., Hoepffner, J., and Schmid, P. J., "Input-Output analysis and control design applied to a linear model of spatially developing flows," Applied Mechanics Reviews, Vol. 62(2), No. $020803,2009$.

[2] Sipp, D., Marquet, O., Meliga, P., and Barbagallo, A., "Dynamics and control of global instabilities in open-flows: a linearised approach," Applied Mechanics Reviews, Vol. 63, No. 030801, 2010.

[3] Efe, M., and Ozbay, H., "Proper orthogonal decomposition for reduced order modelling: 2D heat flow," IEEE Conf. Contr. Appl., Vol. 2, 2003, pp. 1273-1277.

[4] W., R. C., Colonius, T., and Murray, R. M., "Model reduction for compressible flows using POD and Galerkin projection," Physica D, Vol. 189, 2004, pp. 115-129.

[5] Rowley, C. W., "Model reduction for fluids, using balanced proper orthogonal decomposition," International Journal of Bifurcation and Chaos, Vol. 15, No. 3, 2005, pp. 997-1013.

[6] Bagheri, S., Brandt, L., and Nenningson, D. S., "Input/output analysis, model reduction and control of the flat-plate boundary layer," Journal of Fluid Mechanics, Vol. 620, 2009, pp. 263-298.

[7] Akervik, E., Hoepffner, J., Eherenstein, U., and Henningson, D. S., "Optimal growth, model reduction and control in a separated boundary-layer flow using global eigenmodes," Journal of Fluid Mechanics, Vol. 579, 2007, pp. 305-314.

[8] Barbagallo, A., Sipp, D., and Schmid, P., "Closed-loop control of an open cavity flow using reduced-order models," Journal of Fluid Mechanics, Vol. 641, 2009, pp. 1-50.

[9] Hervé, A., Sipp, D., Schmid, J. P., and Samuelides, M., "A physics-based approach to flow control using system identification," Journal of Fluid Mechanics, Vol. 702, 2012, pp. 26-58.

[10] Ljung, L., System identification: theory for the user, $2^{\text {nd }}$ ed., Prentice Hall, 1999.

[11] Huang, S.-C., and Kim, J., "Control and system identification of a separated flow," Physics of Fluids, Vol. 20, No. 101509, 2008.

[12] Sasaki, K., Piantanida, S., Cavalieri, A. V. G., and Jordan, P., "Real-time modelling of wavepackets in turbulent jets," Journal of Fluid Mechanics, Vol. 821, 2017, pp. 458-481.

[13] Sasaki, K., Cavalieri, A. V. G., Silvestre, F., Jordan, P., and Biau, D., "A framework for closed-loop flow control using the parabolized stability equations," Proceedings of the 23rd AIAA/CEAS Aeroacoustics Conference and Exhibit, Denver, United States, 2016.

[14] Sasaki, K., Tissot, G., Cavalieri, A. V. G., Silvestre, F. J., Jordan, P., and Biau, D., "Closed-loop control of a free shear flow: a framawork using the parabolized stability equations," Theoretical and Computational Fluid Dynamics, Vol. 32, 2018, pp. 765-788.

[15] Cavalieri, A. V. G., Jordan, P., Agarwal, A., and Gervais, Y., "Jittering wave-packet models for subsonic jet noise," Journal of Sound and Vibration, Vol. 330, 2011, pp. 4474-4492.

[16] Cavalieri, A. V. G., and Agarwal, A., "Coherence decay and its impact on sound radiation by wavepackets," Journal of Fluid Mechanics, Vol. 748, 2014, pp. 399-415.

[17] Towne, A., "Completing partially known space-time flow statistics: a resolvent-based approach," Tech. rep., Center for Turbulence Research, Annual Review Briefs, 2017.

[18] McKeon, B. J., and Sharma, A. S., "A critical-layer framework for turbulent pipe flow," Journal of Fluid Mechanics, Vol. 658, 2010, pp. 336-382. 
[19] Towne, A., Yang, X., and Lozano-Durán, A., "Approximating space-time flow statistics from a limited set of known correlations,", 2018. Arxiv preprint arXiv:1901.07478v1.

[20] Brès, G., Ham, F. E., Nichols, J. W., and Lele, S. K., "Unstructured alrge-eddy simulations of supersonic jets," AIAA journal, Vol. 55, No. 4, 2017, pp. 1164-1184.

[21] Gudmundsson, K., and Colonius, T., "Instability wave models for the near-field fluctuations of turbulent jets," Journal of Fluid Mechanics, Vol. 689, 2011, pp. 97-128.

[22] Cavalieri, A. V. G., Rodríguez, D., Jordan, P., Colonius, T., and Gervais, Y., "Wavepackets in the velocity field of turbulent jets," Journal of fluid mechanics, Vol. 730, 2013, pp. 559-592.

[23] Jordan, P., and Colonius, T., "Wave Packets and Turbulent Jet Noise," Annual Review of Fluid Mechanics, Vol. 45, 2013, pp. 173-195.

[24] Jordan, P., Zhang, M., Lehnasch, G., and Cavalieri, A. V. G., "Modal and non-modal linear wavepacket dynamics in turbulent jets," Proceedings of the 23rd AIAA/CEAS Aeroacoustics Conference, Denver, United States, 2017.

[25] Bendat, J. S., and Piersol, A. G., Random Data: Analysis and Measurement Procedures, John Wiley \& Sons, 2010.

[26] Rocklin, C. J., G. T, and H., V., "A comparison of $H_{1}, H_{2}$, and $H_{\nu}$ frequency response functions," Proceedings of the 3rd International Modal Analysis Conference, 1985.

[27] Schmidt, O. T., Towne, A., Rigas, G., Colonius, T., and Brès, G. A., "Spectral analysis of jet turbulence," Journal of Fluid Mechanics, Vol. 855, 2018, pp. 953-982.

[28] Semeraro, O., Jaunet, V., Jordan, P., Cavalieri, A. V. G., and Lesshafft, L., "Stochastic and harmonic optimal forcing in subsonic jets," Proceedings of the 21st AIAA/CEAS Aeroacoustics Conference and Exhibit, AIAA, Lyon, France, 2016.

[29] Lesshafft, L., Semeraro, O., Jaunet, V., Cavalieri, A. V. G., and Jordan, P., "Resolvent-baased modelling of coherent wavepackets in a turbulent jet," , 2019. Arxiv preprint arXiv:1810.09340.

[30] Towne, A., Schmidt, O. T., and Colonius, T., "Spectral proper orthogonal decomposition and its relationship to dynamic mode decomposition and resolvent analysis," Journal of Fluid Mechanics, Vol. 847, 2018, p. 821-867. doi:10.1017/jfm.2018.283. 